\title{
Fault Diagnostic System Bearing Centrifugal Pump Using K-Means Method For Thermography Image And Signal Analysis Vibrations
}

\author{
Agung Yudhistira Hidayat ${ }^{1}$, Achmad Widodo $^{2}$, Gunawan Dwi Haryadi ${ }^{1}$ \\ ${ }^{1}$ Departement of Mechanical Engineering Faculty of Engineering, UNDIP, 50275 Semarang, \\ Indonesia
}

\begin{abstract}
Numerous studies reported that infrared thermography and vibration are condition monitoring technology that is important and effective for doing a condition diagnostic of bearing centrifugal pump health without destructing or disturbing machine operational. This paper focuses on thermography image processing based on K-Mean color segmentation which will produce normal and abnormal condition features. Health diagnostic of bearing by processing of digital image, image clustering, segmentation and extraction. Extraction of image pattern is done by calculating the area of heat point and color feature bearing condition of RGB colour space and active contour segmentation in order to process and differentiate between normal and abnormal bearing image by statistical technique. The parameters that can be used as reference to classifying conditions are standard deviation, Mean, Variance, Skewness, Kurtosis, Vibration (RMS) and Shape features (area). Final step is determining the boundary condition between normal and abnormal using statistical logic method.
\end{abstract}

\section{Introduction}

Machine condition monitoring and fault diagnostics become a critical issue in maintenance activity to ensure availability, minimize operator hazard, and reduce economic losses [1]. One of method that is being developed is condition monitoring based on temperature measurement using infrared thermography (IRT). IRT is non-destructive test, free from electromagnetic interference, safe, reliable and can provide large inspection coverage. IRT has taken a very important role for predictive and preventive maintenance programs [2]. Health diagnosis of Machine can be done by evaluating the image pattern generated due to the transformation of input energy into waste heats. IRT can use as a valuable tool for fault diagnosis in Machine which relying on infrared data combined with the heat transfer theory.

Image analysis has many different kinds of methods, one of which is a method of digital-image texture evaluation. The main problem of texture analysis is feature extraction, that extraction allows to explain the characteristics of a digital image numerically.

*Corresponding author: yh_agung@yahoo.co.id 
Statistical methods of the thermal image analysis can be used for monitoring condition and fault diagnosis during machine operation [3]. Segmentation is an important part in image processing before executing the next stage such as feature extraction and classification [4]. Image segmentation methods can be classified into histogram threshold, clustering, edge based, region growing, Physical model-based, Fuzzy approaches and Neural network methods [5]. Clustering techniques identified homogeneous clusters of pixels in the feature space such as RGB, HSV, YIQ, XYZ, LAB, and LUV color space [6][7]. RGB is an additive color system based on the theory of tri-colored (red, green and blue) and nonlinear visual perception.

This paper proposed condition monitoring and health diagnostics of bearing centrifugal pump based on image of thermal infrared and vibration signals. The aim at this research is to find a new alternative source features and a machine diagnostics technique by utilizing thermal images. Thermal images are processed by the threshold method of image segmentation Using K-Means Clustering which will produce a characteristic of normal and abnormal pattern image. Salient features are then extracted from binary images after images processing. This result based on the size (area, perimeter and eccentricity) and color patterns generated on normal and abnormal condition. The effectiveness of thermographic techniques for fault identification of bearing a centrifugal pump is highly dependent on its accuracy in identifying the hot areas and predicting its severity level. Vibration signals analysis used as validation and strengthen the IRT methods Condition monitoring using vibration signal has information on dynamic behavior that is often used for fault detect in this bearing centrifugal pump. Health diagnosis of bearing centrifugal pump is done based on the characteristics thermal image and graph vibration signals measurement results.

\section{Basic Theory}

\subsection{Image Thresholding.}

Image threshold is used for image segmentation [7]. A threshold is a process to separate objects of its background in a digital image. Histogram is the main tool in this separation process. Suppose that the grey level corresponds to an image $f(x, y)$ that is composed of lights objects of a dark background, in such a way that object and background pixels have grey levels grouped into two dominant modes. Both colors are black and white. The colors used for the image are objecting in the foreground. While the rest of the image is the background color. Extracting the object of the background is performed by selecting a threshold $T$ that separates these modes. A threshold image $g(x, y)$ is defined as:

$$
\begin{gathered}
\boldsymbol{g}(\boldsymbol{x}, \boldsymbol{y})=\left\{\begin{array}{c}
0 \text { jika } \boldsymbol{f}(\boldsymbol{x}, \boldsymbol{y})<T, \\
1 \text { jika } \boldsymbol{f}(\boldsymbol{x}, \boldsymbol{y}) \geq T . \\
\text { or }
\end{array}\right. \\
\boldsymbol{g}(\boldsymbol{x}, \boldsymbol{y})=\left\{\begin{array}{c}
0 \text { jika } \boldsymbol{f}(\boldsymbol{x}, \boldsymbol{y}) \geq T, \\
1 \text { jika } \boldsymbol{f}(\boldsymbol{x}, \boldsymbol{y})<T .
\end{array}\right.
\end{gathered}
$$

\subsection{Feature Extraction of image}

Feature extraction is the process to regain the most important data from the raw data to find parameter that defines shape of a character exactly and uniquely [8]. Feature extraction image can be classified into three groups are color feature extraction, shape and texture features [9]. Extraction feature of RGB is example color feature extraction. The calculation of the perimeter and area are meaningful for binary image. Consider a discrete binary image 
containing one more object, where $\mathbf{p}(\mathrm{x}, \mathrm{y})=1$ if a pixel is part of the object or foreground and $\mathbf{p}(\mathrm{x}, \mathrm{y})=0$ for background pixels $[10]$.

\section{Methodology}

\subsection{Data acquisition process}

The first task supposedly begins with the image acquisition process. The experimental setups that were carried out by using thermographic camera FLIR E-40 at a distance of 2 metres, measure the temperature on a range scale of $-20{ }^{\circ} \mathrm{C}$ to $650{ }^{\circ} \mathrm{C}$, The emissivity value was set at 0.95 as recommended material of cast iron [11], ambient temperature is $30-41{ }^{\circ} \mathrm{C}$. The experiment was carried out with the condition of centrifugal pump operation full load in the power generation unit and the condition of bearing is in normal state.Vibration signals data taken with vibscanner. Measurements are made on bearing of centrifugal pump with specifications: 1,000 HP/Type Horizontal Centrifugal, bearing SKF Explorer 6310-2Z and bearing SKF Explorer 6207.

The result of vibration measurement is Root-Mean Square (RMS in $\mathrm{mm} / \mathrm{s}$ ) data record, while Flir E-40 produces thermal images 320 x 240 pixels. After image acquisition from the object, thermal image is cropped to select the observed region of interest (ROI) in MATLAB. The image acquisition processed for normal and abnormal conditions was then conducted.

\subsection{Image Segmentation}

Segmentation image thermography is done to decompose a thermal image of specific parts of further analysis, resulting in a higher level representation of the image pixels like objects and background image [12]. One of the image segmentation methods is clustering, clustering techniques identified homogeneous clusters of pixels in the feature space such as RGB color space [5]. The pixel features of images are clustered using the K-Means clustering algorithm to group them into five regions of similar color and selected one image of ROI representing temperature increases that is redness image region. This ROI calculates to do RGB characteristic (Mean, standard deviation, kurtosis, skewness and entropy.) and shape feature (area, perimeter and eccentricity).
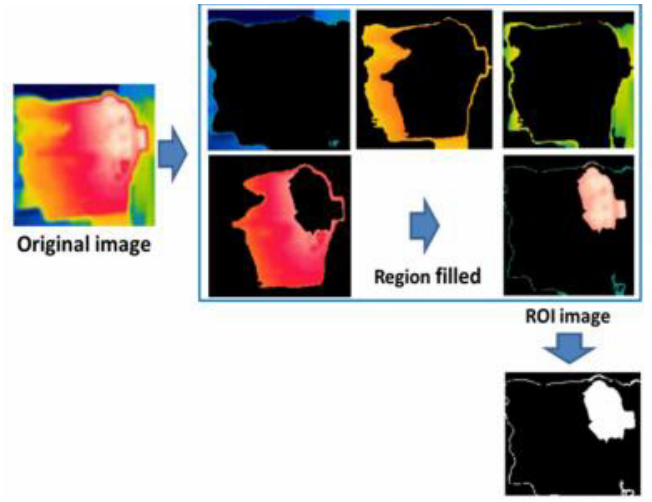

RGB observable

(a)

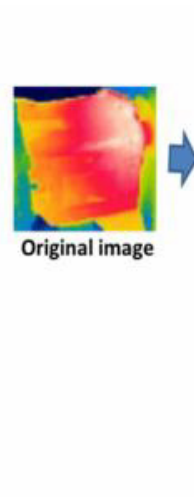

(b)

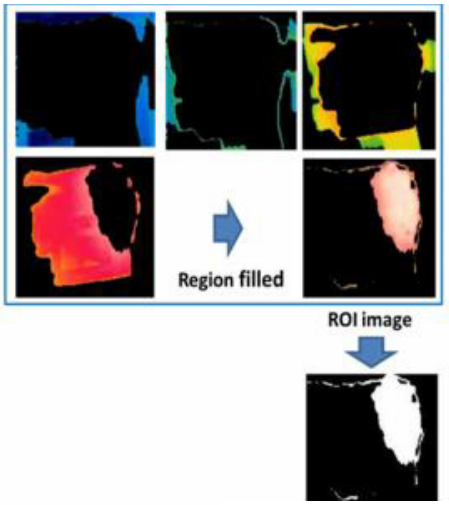

RGB observable

Fig.1. Color segmentation process, decide to ROI: (a) Normal condition, (b) Abnormal condition 


\subsection{Image feature extraction}

Clustering process will produce one color image area that will be implemented image analysis, calculation of RGB characteristic (Mean, standard deviation, kurtosis, skewness, variance and entropy.), shape feature (area, perimeter and eccentricity) and vibration. Image statistics are investigated and studied based on first and higher order statistics that capture certain statistical regularities of ROI thermal image [7], following equation:

$$
\begin{gathered}
\text { Mean }(\mu)=\frac{1}{M x N} \sum_{x=1}^{M} \sum_{y=1}^{N} p(x, y), \text { Std.dev. }(\sigma)=\sqrt{\frac{1}{M x N} \sum_{x=1}^{M} \sum_{y=1}^{N}(p(x, y)-\mu)^{2}} \\
\text { Entropy }\left(E_{n}\right)=\frac{1}{M x N} \sum_{x=1}^{M} \sum_{y=1}^{N} p(x, y) \log _{2}(p(x, y)) \\
\text { Skewness }(s)=-\frac{1}{M x N} \sum_{x=1}^{M} \sum_{y=1}^{N}\left|\frac{p(x, y)-\mu}{\sigma}\right|^{3} \\
\text { Kurtosis }(k)=-\frac{1}{M x N} \sum_{x=1}^{M} \sum_{y=1}^{N}\left|\frac{p(x, y)-\mu}{\sigma}\right|^{4}-3
\end{gathered}
$$

Next step is plotting the features of statistical RGB and shape feature. Afterwards selected features that can distinguish between normal and abnormal conditions. Final step is determining the boundary condition between normal and abnormal using statistical logic method. Boundary values obtained by calculation value of mean, min, max, standard deviation, delta (D) of selected feature data. Value of the delta (D) is average difference between minimum and maximum value in each class.

$$
\text { final } \max =\max +D, \text { final } \min =\min -D
$$

\section{Result and Discussion}

A sentrifugal pump being subjected to this observation according to ISO 10816-3 can be categorized into the group one rigid. Classification based on motor power is more than 300 KW. Normal condition if the root mean square (RMS) values has up $7.1 \mathrm{~mm} / \mathrm{s}$. If then RMS $\geq 7.1 \mathrm{~mm} / \mathrm{s}$ been categorized as abnormal condition. From number 50 data can be classified into number 40 data onto normal condition and 10 data of abnormal condition. The acquire images stored in RGB format with size of $256 \times 256$ pixels. The ROI was determined by red or white colors that represent the hottest temperature area generated by bearings and will be transformed into binary image by threshold method, then extracted to calculate area, perimeter and eccentricity.

The ROI image determination process image clustering are presented figure 1 . ROI is an RGB image, extracted to calculate the statistical of each component of RGB. Referring to determination and the resulting feature, not all features can be used as reference to differentiate of bearing centrifugal pump conditions. Figure 2 shows random patterns for the features that can differentiate its condition are standard deviation, mean, variance, skewness, kurtosis of RGB. 

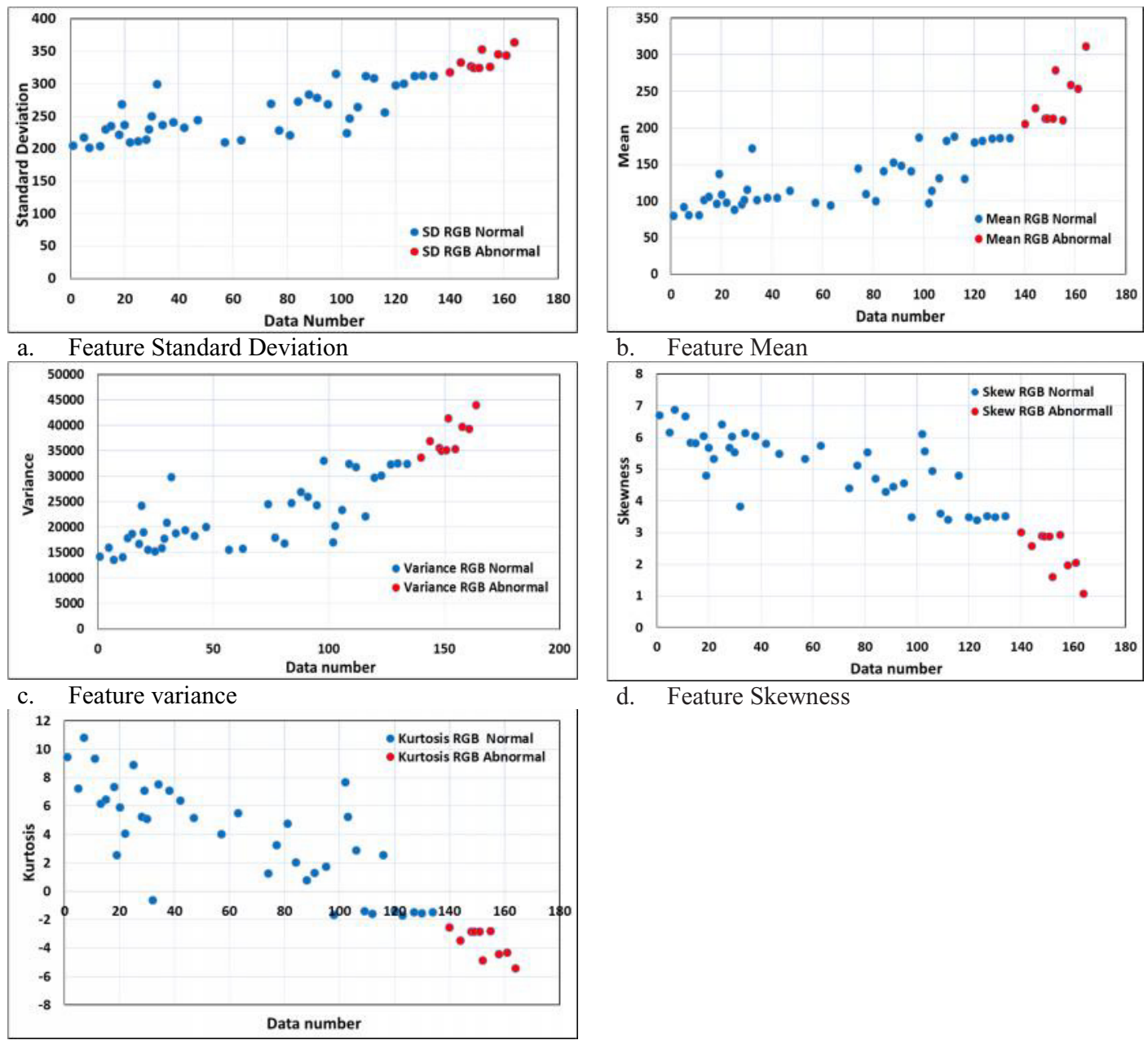

d. Feature Skewness

e. Feature Kurtosis

Fig. 2. Feature differentiate between normal and abnormal condition

Based on the result of calculation of equation 6 in Table 1, determination of boundary conditions value (Final maximum/F. Max and minimum/F. Min) does not have same value of boundary condition between normal and abnormal condition.

Table 1. Determination result of boundary condition value of each parameter RGB.

\begin{tabular}{|c|c|c|c|c|c|c|c|c|c|c|c|c|}
\hline \multirow{2}{*}{$\begin{array}{l}\text { Condition of Bearing } \\
\text { Centrifugal Pump }\end{array}$} & \multicolumn{2}{|c|}{ RMS } & \multicolumn{2}{|c|}{ SD } & \multicolumn{2}{|c|}{ Mean } & \multicolumn{2}{|c|}{ Variance } & \multicolumn{2}{|c|}{ Skewness } & \multicolumn{2}{|c|}{ Kurtosis } \\
\hline & Min & Max & Min & Max & Min & Max & Min & Max & Min & Max & Min & Max \\
\hline Normal & 1,83 & 89 & 00,82 & 314,77 & 79,56 & 187,81 & 13578,76 & 33088,85 & 3,39 & 6,87 & $-1,70$ & 10,80 \\
\hline bnormal & 7,17 & 9,63 & 317,48 & 363,16 & 204,73 & 311,03 & 33734,32 & 43987,12 & 1,07 & 3,00 & $-5,43$ & $-2,53$ \\
\hline
\end{tabular}

\section{Conclusion}

Based on this study, color segmentation on the thermal image can be used to diagnose normal and abnormal conditions on the bearing of centrifugal pump. Parameters that can be used a good reference to distinguish between normal and abnormal conditions are standard 
deviation, Mean, Var, Skewness, Kurtosis. Bearing on centrifugal pump can be categorized abnormal if RMS $>7.17 \mathrm{~mm} / \mathrm{s}$, standard deviation $\geq 317.48$, Mean $\leq 204.73$, Var $\geq$ $33,734.32$, Skewness $\leq 3$ and Kurtosis $\leq-2.53$.

The author gratefully acknowledges the support of measuring instruments and research objects of PT. Indonesia Power Semarang on this research.

\section{References}

1. Lei, Y., He, Z., \& Zi, Y. Application of an intelligent classification method to mechanical fault diagnosis. Expert Systems with Applications, 36(6), 9941-9948. (2009).

2. Cao, Y., Gu, X., \& Jin, Q. Infrared technology in the fault diagnosis of substation equipment. In Electricity Distribution, 2008. CICED 2008. China International Conference on (pp. 1-6). IEEE. (2008, December).

3. Fidali, M., An idea of continuous thermographic monitoring of machinery, QIRT 2008 Proceedings, In 9th International Conference on Qualitative Infrared Thermography, pp. 61-68. (2008).

4. Solomon, C., \& Breckon, T.,, Fundamentals of Digital Image Processing: A practical approach with examples in Matlab, John Wiley \& Sons. (2011).

5. Chauhan, Poonam, and R. V. Shahabade, Mean shift segmentation on Enhanced Color image, IOSR Journal of Computer Engineering (IOSR-JCE) e-ISSN: 2319-8753, Vol.3, (2014).

6. Wang, H., \& Suter, D., Color image segmentation using global information and local homogeneity, In Proceeding of 7th Conf. of Digital Image Computing: Techniques and Applications. (2003).

7. Ravichandran, D., R. Nimmatoori, and M. R. Ashwin Dhivakar. "A study on Image Statistics and Image Features on Coding Performance of Medical Images." Int. J. of Advanced Computer Engineering and Communication Technology (IJACECT) 5,1, p.16 (2016).

8. R. C. Gonzalez and R. E. Woods, Digital Image Processing, Prentice-Hall, Upper Saddle River, NJ, USA, 2nd edition, (2002).

9. Kumar, Gaurav, and Pradeep Kumar Bhatia. "A detailed review of feature extraction in image processing systems." Advanced Computing \& Communication Technologies (ACCT), 2014 Fourth International Conference on. IEEE, pp. 5-12, (2014).

10. Medjahed, Seyyid Ahmed. "A Comparative Study of Feature Extraction Methods in Images Classification." International Journal of Image, Graphics and Signal Processing 7.3: 16, (2015)

11. Bagavathiappan, S., Lahiri, B. B., Saravanan, T., Philip, J., \& Jayakumar, T., Infrared thermography for condition monitoring-a review, Infrared Physics \& Technology, Volume 60, pp. 35-55, (2013).

12. Sural, Shamik, Gang Qian, and Sakti Pramanik. "Segmentation and histogram generation using the HSV color space for image retrieval." Image Processing, Proceedings. 2002 International Conference on. Vol. 2. IEEE (2002). 